\title{
Review: studies on the cardiovascular effects of selective COX-2 inhibitors show mixed results
}

Mukherjee D, Nissen SE, Topol EJ. Risk of cardiovascular events associated with selective COX-2 inhibitors. JAMA 2001

Aug 22/29;286:954-9.

\section{QUESTION: In patients with arthritis, do rofecoxib or celecoxib increase the risk for cardiovascular events?}

\section{Data sources}

Studies were identified by searching Medline (1998 to February 2001) and the internet. The Adverse Events Reporting System was searched for US events on October 12, 2000.

\section{Study selection}

English language studies were selected if they were randomised, double blind, controlled trials reporting the cardiovascular effects of celecoxib or rofecoxib.

\section{Data extraction}

Data were extracted on study methods, patient characteristics, drug regimens, aspirin use, and cardiovascular events.

\section{Main results}

4 studies met the selection criteria. The Vioxx Gastrointestinal Outcomes Research study compared rofecoxib $50 \mathrm{mg} /$ day, with naproxen $1000 \mathrm{mg} /$ day, in 8076 patients with rheumatoid arthritis. Aspirin use was not permitted. Patients in the rofecoxib group had an increased risk for cardiovascular events \{relative risk increase 138\%, 95\% CI 39 to 300; number needed to harm 146, CI 69 to 517\}*. The Celecoxib Long-term Arthritis Safety Study (CLASS) compared celecoxib 400 $\mathrm{mg}$ twice daily; ibuprofen $800 \mathrm{mg} 3$ times daily; and diclofenac $75 \mathrm{mg}$ twice daily, in 8059 patients with osteoarthritis or rheumatoid arthritis. Patients were allowed to take aspirin $(<325 \mathrm{mg} /$ day). Celecoxib and non-steroidal anti-inflammatory drugs did not differ for cardiovascular event rates. 2 studies compared rofecoxib $12.5 \mathrm{mg} /$ day; nabumetone $1000 \mathrm{mg}$ /day; and placebo for 6 weeks in 1042 and 978 patients with osteoarthritis of the knee. Patients were allowed to take low dose aspirin. In both studies, the groups did not differ for cardiovascular event rates.

\section{Conclusions}

Compared with other non-steroidal anti-inflammatory drugs (NSAIDs), highly cyclooxygenase-2-selective NSAIDs seem to increase the risk for cardiovascular thrombotic events in patients who are not taking aspirin.

*Numbers calculated from data in article

\section{COMMENTARY}

Platelet cyclooxygenase (COX)-1 is a main source of the potent vasoconstrictor and platelet aggregator $\mathrm{TxA}_{2}$. Macrovascular endothelial COX-2 is a main source of the potent vasodilator and platelet inhibitor $\mathrm{PGI}_{2} \cdot{ }^{1}$ Thus, one might well have predicted that the highly COX-2-selective inhibitor, rofecoxib, would increase the risk for thrombosis. In an ex vivo assay in human whole blood, celecoxib was less COX-2-selective than rofecoxib, etodolac, or meloxicam, and only slightly more selective than nimesulide. ${ }^{2}$

The clinical research to date confirms the available basic research. Compared with non-selective NSAIDs, rofecoxib slightly decreases the risk for gastrointestinal (GI) haemorrhage but increases the risk for thrombosis. The most important celecoxib study (CLASS) ${ }^{1}$ is now mired in controversy because of the publication of the more favourable 6 month data instead of the less favourable 12 month outcomes. Celexicob's failure to decrease GI events and failure to increase vascular events relative to ibuprofen and diclofenac could be explained by either the co-administration of aspirin allowed in CLASS or by its relatively modest COX-2 selectivity.

The concomitant use of aspirin, even at low doses, may largely negate the GI safety advantage of COX-2-selective NSAIDs Additionally, some NSAIDs (eg, ibuprofen) seem to inhibit the protective effects of aspirin, whereas others (eg, diclofenac and rofecoxib) do not. ${ }^{4}$ The current well supported and widespread use of aspirin may increase depending on the results of the Aspirin in Asymptomatic Atherosclerosis Trial and other studies currently being done.

Physicians should continue to prescribe aspirin for secondary prevention of vascular events, with the understanding that patients who need aspirin will probably derive no safety advantage from COX-2-selective NSAIDs. Until additional pharmacodynamic studies and large clinical trials determine the clinical importance of its prothrombotic effects, rofecoxib should be avoided. Steven Belknap, MD University of Illinois College of Medicine at Peoria Peoria, Illinois, USA

1 McAdam BF, Catella-Lawson F, Mardini IA, et al. Systematic biosynthesis of prostacyclin by cyclooxygenase (COX)-2: the human pharmacology of a selective inhibitor of COX-2. Proc Natl Acad Sci USA 1999;96:272-7.

2 Warner TD, Giuliano F, Vojnovic I, et al. Nonsteroid drug selectivities for cyclo-oxygenase-1 rather than cyclo-oxygenase 2 are associated with human gastrointestinal toxicity: a full in vitro analysis. Proc Natl Acad Sci USA 1999;96:7563-8.

3 CLASS Advisory Committee Briefing Document. http://www.fda.gov/ohrms/dockets/ac/01/briefing/3677b1_01_searle.pdf.

4 Catella-Lawson F, Reilly MP, Kapoor SC, et al. Cyclooxygenase inhibitors and the antiplatelet effects of aspirin. $N$ Engl J Med 2001;345:1809-17. 